\title{
MASTOFAUNA DEL ÁREA DE PROTECCIÓN DE FLORA Y FAUNA LA PRIMAVERA, JALISCO, MÉXICO
}

\author{
Silvia S. ZALAPA, ${ }^{1 *}$ Edgar G. GODINEZ ${ }^{2}$ \& SERGIo GUERRERO ${ }^{1}$ \\ ${ }^{1}$ Centro de Estudios en Zoología, Universidad de Guadalajara. Av. Aviación 4055, Milán 14, San Juan \\ de Ocotan, Zapopan, Jalisco, México. CP 45019. <sszalapah@gmail.com>, <sergioguerrerovazquez@ \\ gmail.com> \\ ${ }^{2}$ Departamento de Zoología, Instituto de Biología, Universidad Nacional Autónoma de México, Ap. \\ Postal 70-153, México, D. F. <eggodinez@gmail.com>.
}

Zalapa, S. S., Godinez, E. G. \& Guerrero, S. 2014. Mastofauna del área de protección de flora y fauna La Primavera, Jalisco, México. Acta Zoológica Mexicana (n.s.), 30(1): 18-31.

RESUMEN. El Área de Protección de Flora y Fauna La Primavera (APFFLP) se localiza en el occidente de México en el estado de Jalisco, es una de las 19 áreas protegidas decretadas en la entidad. Esta área es de gran interés al ubicarse en la Zona Metropolitana de la Ciudad de Guadalajara y en la Faja Volcánica Transmexicana. Sin embargo, ha recibido poca atención y a la fecha no existe un inventario completo de su mastofauna. El objetivo de este trabajo es listar las especies de mamíferos presentes en el APFFLP, y evaluar su relevancia con relación a otras áreas naturales protegidas en la entidad y del país, y su riqueza dentro de la Faja Volcánica Transmexicana. El trabajo de campo se llevó a cabo en dos periodos, diciembre 2004-diciembre 2006 y octubre 2010-diciembre 2012. En el primero se usaron diversas técnicas como capturas y registros visuales de los mamíferos, excepto murciélagos; en el segundo se usaron redes de niebla para la recolecta de la quiropterofauna. Se registraron 59 especies, las cuales quedan comprendidas en ocho órdenes, 19 familias y 48 géneros. El orden Chiroptera fue el más abundante (27), seguido de Rodentia (14) y Carnivora (11). En relación con su distribución geográfica actual, 23 especies se consideraron de amplia distribución, 17 compartidas con Norteamérica, 10 endémicas a México y Mesoamérica y nueve compartidas con América del Sur. De acuerdo con la legislación mexicana, se registran tres especies amenazadas (Cratogeomys fumosus, Leptonycteris yerbabuenae y Choeronycteris mexicana). En conclusión, resalta su importancia por albergar más especies y endemismos que otras áreas consideradas relevantes en el país, además de ser un área importante de riqueza de mamíferos en la Faja Volcánica Transmexicana.

Palabras clave: bosque de encino-pino, Guadalajara, Faja Volcánica Transmexicana, murciélagos, roedores.

Zalapa, S. S., Godinez, E. G. \& Guerrero, S. 2014. Mammals of the protection area of flora and fauna La Primavera, Jalisco, Mexico. Acta Zoológica Mexicana (n.s.), 30(1): 18-31.

ABSTRACT. The Protection Area of Flora and Fauna La Primavera (APFFLP) located in western Mexico in the state of Jalisco, is one of 19 protected areas in the state decreed. This area is of great inter-

Recibido: 22/08/2012; aceptado: 10/09/2013. 
est to be located in the metropolitan area of the city of Guadalajara and the Transmexican Volcanic Belt. However, it has received little attention and there is not a full inventory of its mammalian fauna. The aim of this paper is to list the species of mammals in the APFFLP, highlight its relevance to other protected areas in the state and the country, and richness within the Transmexican Volcanic Belt. Fieldwork was conducted in two periods, December 2004-December 2006, and October 2010-December 2012. The checklist of mammals was obtained using several techniques such as traps and visual records of the mammals and tracks; we used mist nets for the collection of bats. 59 species were recorded, which are covered in eigth orders, 19 families and 48 genera. The order Chiroptera was the most abundant (27), followed by Rodentia (14) and Carnivora (11). The distribution of species shows that 23 are shared with North and South America; 17 with North America; nine with South America and 10 are endemic to Mexico and Meso America. Three species are considered at risk by Mexican regulations: Cratogeomys fumosus, Leptonycteris yerbabuenae and Choeronycteris mexicana as threatened. In conclusion, APFFLP is important for harboring more species and endemic species than other areas considered relevant in the country, besides being an important area of mammal species richness in the Transmexican Volcanic Belt.

Key words: Guadalajara, Transmexican Volcanic Belt, oak-pine forest, bats, rodents.

\section{INTRODUCCIÓN}

El establecimiento de Áreas Naturales Protegidas (ANPs) es una de las principales estrategias a nivel internacional y nacional para la conservación de la diversidad biológica (Hockings 2003, Ceballos 2007, CONABIO 2007). En México existen actualmente 176 ANPs federales, las cuales abarcan aproximadamente el 12\% del territorio nacional (CONANP 2012). En el estado de Jalisco se han decretado 19 ANPs lo cual corresponde aproximadamente al 10\% del área total en esa entidad (SEMADET 2013). Sin embargo, muchas de estas ANPs ven comprometida su eficacia como áreas de conservación, debido a diversas problemáticas, una de ellas es el limitado conocimiento de su riqueza biológica (Cervantes et al. 1995). Se reconoce que en México más de 80 ANPs carecen de esta información (Ceballos 2007); incluso es un problema que enfrentan muchas ANPs en el mundo (Hockings 2003).

Se tiene una idea clara de la riqueza de mamíferos que alberga el estado de Jalisco (Iñiguez \& Santana 1993, Guerrero \& Cervantes 2003, Ramos-Vizcaíno et al. 2007, Godinez et al. 2011), sin embargo a nivel regional y local no es el caso, lo cual se evidencia en las ANPs de la entidad. De las 19 áreas con decreto, solo se cuenta con listas de mamíferos actualizadas de la Reserva de la Biosfera Chamela-Cuixmala, en las cuales se citan 70 especies (Miranda 2002). Para otras ANPs, se cuenta con listados parciales, tal es el caso de la Reserva de la Biosfera Sierra de Manantlán en la que se reconocen 110 especies (INE 2000), pero sólo tienen publicadas listas de especies endémicas o con categoría de protección con 25 especies (INE 2000), murciélagos con 55 (Iñiguez 1993) y un estudio sobre roedores en el que se cita la presencia de siete especies (Vázquez et al. 2000). También es el caso del Complejo Volcánico de Colima con una extensión de $843.13 \mathrm{~km}^{2}$, en dónde se encuentra incluido el Parque Nacional Volcán Nevado de Colima, que sólo cuenta con el registro de 25 especies 
de murciélagos (Segura-Trujillo \& Navarro-Pérez, 2010). Asimismo para la zona de Arcediano (ubicada al noreste de la ciudad de Guadalajara, dentro de la cuenca del Río Santiago y Río Verde) se incluyen 53 especies de mamíferos (Godinez et al. 2008). Aún menos información se conoce sobre el Área de Protección de Flora y Fauna La Primavera (APFFLP), donde Juárez-Aguilar (1995) registró 13 especies de mamíferos y en el plan de manejo de esta área se reconocen solamente 29 (CONANP 2000).

La eficiencia de las ANPs en la conservación de los mamíferos en México ha sido evaluada, en función de su riqueza, especies en riesgo, endemismos y/o rangos de distribución. En este sentido, la relevancia del APFFLP ha sido considerada mínima ubicándola en el lugar número 95 de 136 (Escalante 2003), mientras que en otras evaluaciones no fue incluida (Fa \& Morales 1991, Ceballos 2007, Vázquez \& Valenzuela-Galván 2009). Sin embargo, se ha reconocido su relevancia por su extensión, en relación a otras ANPs presentes en la Faja Volcánica Trasnmexicana (FVT), tomando el lugar número ocho de 39 (Fuller 2006). En otro estudio en el que se usaron modelos de distribución potencial, superpuestos a una cuadricula de $0.5^{\circ} \mathrm{x}$ $0.5^{\circ}$ se predijo para la celda donde se ubica el APFFLP una riqueza de 57 especies de mamíferos (Escalante et al. 2007).

En el APFFLP se desarrollan importantes procesos ecológicos como la captación, filtración, transporte y saneamiento de aguas superficiales, así como regulación del clima y protección a cuencas y subcuencas hidrológicas, que representan uno de los principales aportes de agua a la región (CONANP 2000). Forma parte de la FVT la cual es reconocida por su alta riqueza y numerosos endemismos de mamíferos (Ceballos \& Navarro 1991, Escalante 2007) conteniendo 12 de los 13 géneros de éste grupo endémicos a México (Gámez et al. 2012), también se considera centro de diversificación y transición biogeográfica (Ramamoorthy et al. 1998, Morrone 2005). Entre algunas de las problemáticas que amenazan la biodiversidad de la FVT están la deforestación y el incremento de las actividades humanas (Ceballos \& Navarro 1991, Velázquez et al. 2002); situaciones a las que se enfrenta el APFFLP. Con base en lo anterior, el objetivo de este trabajo es listar las especies de mamíferos presentes en el APFFLP y evaluar su relevancia en relación a la FVT y otras áreas del estado y del país.

\section{MATERIALES Y MÉTODOS}

Zona de estudio. El APFFLP se localiza en la región central del estado de Jalisco, México, al poniente de la ciudad de Guadalajara (103²8' N y $20^{\circ} 32^{\prime} \mathrm{O}$ ), cuenta con una extensión de 30,500 has (Fig. 1). El clima predominante es templado subhúmedo $\mathrm{S}\left(\mathrm{w}_{1}\right)(\mathrm{w})$ y semicálido subhúmedo $(\mathrm{A}) \mathrm{C}\left(\mathrm{w}_{1}\right)(\mathrm{w})$, ambos con lluvias en verano e invierno; las precipitaciones anuales fluctúan entre 800 y $1,000 \mathrm{~mm}$, siendo las invernales sólo del $5 \%$ de la precipitación total. La temperatura media anual es de $20{ }^{\circ} \mathrm{C}$ $\left( \pm 6.5^{\circ} \mathrm{C}\right)$, y el promedio anual de humedad es de $63 \%$ (CONANP 2000 ). 

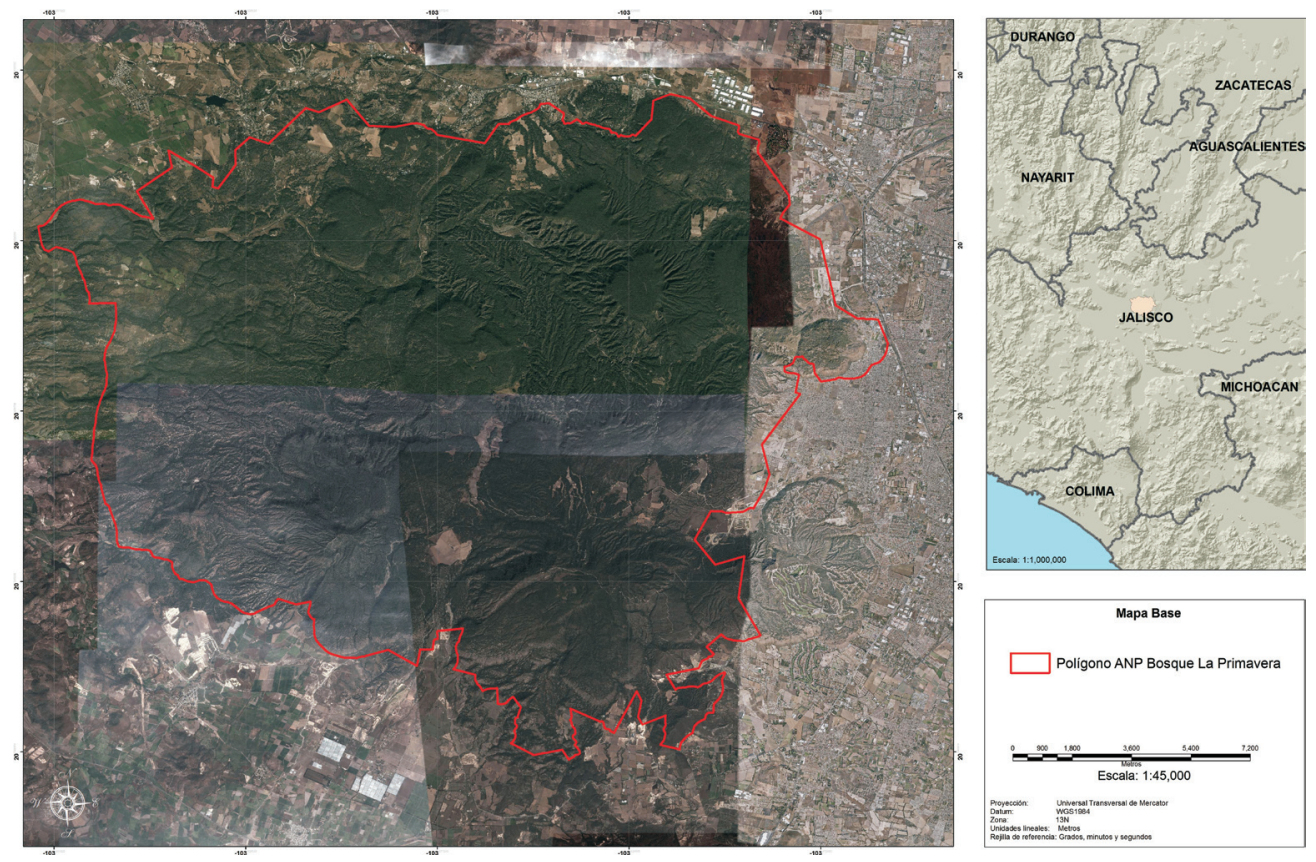

Figura 1. Ubicación del Área de Protección de Flora y Fauna La Primavera, Jalisco, México.

La diversidad vegetal está influenciada por el traslape de dos provincias florísticas: Sierra Madre Occidental y Eje Neovolcánico Transversal. A su vez, estas provincias se localizan en la Región Mesoamericana de Montaña, formada por la confluencia de dos grandes regiones geográficas la Neártica y la Neotropical (CONANP 2000). De acuerdo con la clasificación de Rzedowski (1978) se encuentran cuatro tipos de vegetación: bosque de encino, encino-pino, pino, y bosque tropical caducifolio. La vegetación más representativa es el bosque de encino-pino, el cual se encuentra desde los 1,800 hasta los 2,225 metros sobre el nivel del mar (msnm), en los puntos más altos del área conocidos como cerro San Miguel y cerro Planillas. Los elementos arbóreos alcanzan alturas de 6 a $15 \mathrm{~m}$, considerando a Quercus castanea, Q. laeta, Q. obtusata, $Q$. coccolobifolia, $Q$. viminea, $Q$. magnoliifolia, Pinus oocarpa, P. douglasiana, Clethra rosei, Agarista mexicana y Prunus serotina var. capui los predominantes (CONANP 2000).

El registro de las especies de mamíferos se basó en trabajo de campo, ejemplares depositados en la colección del Centro de Estudios en Zoología de la Universidad de Guadalajara (CEZUG) y análisis de literatura compilada. El trabajo de campo se llevó a cabo en dos periodos. El primero diciembre 2004- diciembre 2006, se visitaron diferentes sitios y se usaron diversas técnicas de muestreo (trampas Sherman, pitfall, 
y trampas para la captura de tuzas (Geomyidae) vivas e interpretación de rastros y observaciones directas). Se utilizaron 25 trampas tipo Sherman, en transecto lineal, separadas cada una por $10 \mathrm{~m}$ y colocadas en un sitio por noche durante tres días consecutivos, en 10 sesiones de muestreo, para un total de 750 noches trampa. Las trampas se cebaron con una mezcla de avena y vainilla y se revisaron en la mañana siguiente. Además se realizaron recorridos tanto diurnos como nocturnos en el área de estudio y su zona de influencia durante los días de muestreo para la interpretación de rastros (huellas y excretas). Para la identificación de los rastros se tomó la referencia de Aranda (2000). En el segundo periodo octubre 2010- diciembre 2012, se recolectaron murciélagos, utilizando 10 redes de niebla $(12 \times 2.6 \mathrm{~m})$ en tres sitios diferentes, una noche por sitio por mes; las redes se activaron durante cinco horas a partir del ocaso, con un total de $82,680 \mathrm{~m}^{2}$ red hora. En cada sitio se ubicaron cinco redes sobre un cuerpo de agua y cinco entre la vegetación. Los especímenes registrados en campo fueron determinados hasta nivel de especie, basándose en literatura especializada (Jones et al. 1970, Hall 1981, Kumirai \& Jones 1990, Álvarez et al. 1994, Medellín et al. 2008).

Para confirmar las determinaciones, se realizaron recolectas de ejemplares los cuales se prepararon como ejemplares de museo y se depositaron en la colección de vertebrados del CEZUG, en la ciudad de Guadalajara, Jalisco (permiso especial de recolecta científica SGPA/DGVS/06167/10 y SGPA/DGVS/01849/12). Las fotografías de ejemplares no recolectados se depositaron en la colección fotográfica de mamíferos del CEZUG.

Los endemismos fueron considerados acorde a lo propuesto por Ramírez-Pulido et al. (2005) y las categorías de riesgo de extinción se basaron en la NOM-059-SEMARNAT-2010 (SEMARNAT 2010). Para la categoría de distribución geográfica actual se siguió la propuesta por Ceballos et al. (2002) y la nomenclatura y arreglo taxonómico utilizados fueron según Godinez et al. (2011).

Para comparar la riqueza de mamíferos del APFFLP con otras ANPs del país, se calculó el número de especies por $10 \mathrm{~km}^{2}$, se usó el listado de ANPs consideradas por Ceballos (2007), del cual solo se incluyeron las ANPs con el mismo bioma (bosque templado de coníferas) y de mayor extensión que el APFFLP. De las ANPs de Jalisco se consideraron aquellas que cuentan con listados actualizados y de la FVT el trabajo de Escalante et al. (2007).

\section{RESULTADOS}

Se registraron 59 especies las cuales quedan comprendidas en ocho órdenes, 19 familias y 48 géneros (Cuadro 1). Los registros del ratón tlacuache (Tlacuatzin canescens), pecarí de collar (Tayasu tajacu) y puma (Puma concolor) fueron obtenidos por personal del APFFLP, mediante observación directa y fotografía en cámaras trampa. El orden con mayor número de especies fue Chiroptera (murciélagos) con 27, seguido 
Cuadro 1. Mamíferos registrados en el Área de Protección de Flora y Fauna La Primavera, Jalisco, México. Se siguió el arreglo nomenclatural y taxonómico según Godinez et al. (2011). Distribución (distribución geográfica actual): MA (endémica a México y Mesoamérica), AM (compartida con Norte y Sur América), NA (compartida con Norteamérica) y SA (compartida con América del Sur). Endémica de México: EM. NOM-059: (Categoría en la NOM-059-SEMARNAT-2010): A (Amenazada). Medio de registro: cap = capturado durante este estudio; col = colectado durante este estudio y depositado en la Colección de Vertebrados del CEZUG; foto: foto depositada en la colección fotográfica de mamíferos del CEZUG; lit = literatura, fuente Juárez-Aguilar 1995; CZUG = registro de la Colección de Vertebrados del CEZUG; obs = observación directa en campo; rastro = huellas y/o excretas; DVSLP = registro realizado por la Dirección de Conocimiento y Vida Silvestre del APFFLP, mediante cámara trampa u observación directa.

\begin{tabular}{|c|c|c|c|c|}
\hline & Distribución & Endemismo & NOM-059 & $\begin{array}{l}\text { Medio de } \\
\text { registro }\end{array}$ \\
\hline \multicolumn{5}{|l|}{ DIDELPHIMORPHIA } \\
\hline \multicolumn{5}{|l|}{ DIDELPHIDAE } \\
\hline Didelphis virginiana Kerr, 1972 & $\mathrm{AM}$ & & & lit/obs/foto \\
\hline Tlacuatzin canescens (J.A. Allen, 1893) & MA & EM & & DVSLP \\
\hline \multicolumn{5}{|l|}{ CINGULATA } \\
\hline \multicolumn{5}{|l|}{ DASYPODIADE } \\
\hline Dasypus novemcintus Linnaeus, 1758 & $\mathrm{AM}$ & & & lit/obs \\
\hline \multicolumn{5}{|l|}{ RODENTIA } \\
\hline \multicolumn{5}{|l|}{ SCIURIDAE } \\
\hline Ictidomys mexicanus (Erxleben, 1777) & NA & & & obs \\
\hline $\begin{array}{l}\text { Otospermophilus variegatus (Erxleben, } \\
\text { 1777) }\end{array}$ & NA & & & obs \\
\hline Sciurus nayaritensis J.A. Allen, 1890 & NA & & & obs \\
\hline \multicolumn{5}{|l|}{ HETEROMYIDAE } \\
\hline Liomys irroratus (Gray, 1868) & NA & & & CZUG/cap \\
\hline \multicolumn{5}{|l|}{ GEOMYIDAE } \\
\hline Cratogeomys fumosus (Merriam, 1894) & MA & EM & A & cap/foto \\
\hline Pappogeomys bulleri (Thomas, 1892) & MA & EM & & col \\
\hline \multicolumn{5}{|l|}{ CRICETIDAE } \\
\hline Baiomys taylori (Thomas, 1887) & NA & & & $\mathrm{col} /$ foto \\
\hline Neotoma mexicana Baird, 1855 & NA & & & cap/foto \\
\hline Peromyscus sp. (grupo boylii)* & & EM & & cap/foto \\
\hline Peromyscus maniculatus (Wagner, 1845) & NA & & & $\mathrm{col} /$ foto \\
\hline Peromyscus spicilegus J.A. Allen, 1897 & MA & EM & & cap/foto \\
\hline Reithrodontomys fulvescens J.A. Allen, 1894 & NA & & & $\mathrm{col} /$ foto \\
\hline Sigmodon hispidus Say y Ord, 1825 & $\mathrm{AM}$ & & & col \\
\hline
\end{tabular}


Cuadro 1. Continúa.

\begin{tabular}{|c|c|c|c|c|}
\hline & Distribución & Endemismo & NOM-059 & $\begin{array}{l}\text { Medio de } \\
\text { registro }\end{array}$ \\
\hline Sigmodon mascotensis J.A. Allen, 1897 & MA & EM & & cap/foto \\
\hline \multicolumn{5}{|l|}{ LAGOMORPHA } \\
\hline \multicolumn{5}{|l|}{ LEPORIDAE } \\
\hline Sylvilagus floridanus (J.A. Allen, 1890) & AM & & & obs/rastros \\
\hline \multicolumn{5}{|l|}{ SORICOMORPHA } \\
\hline \multicolumn{5}{|l|}{ SORICIDAE } \\
\hline Cryptotis parva (Say, 1823) & AM & & & obs \\
\hline \multicolumn{5}{|l|}{ CHIROPTERA } \\
\hline \multicolumn{5}{|l|}{ PHYLLOSTOMIDAE } \\
\hline Anoura geoffroyi Gray, 1838 & SA & & & $\mathrm{col} /$ foto \\
\hline Artibeus hirsutus Andersen, 1906 & MA & EM & & $\mathrm{col} /$ foto \\
\hline Artibeus jamaicensis Leach, 1821 & SA & & & $\mathrm{col} /$ foto \\
\hline Artibeus lituratus (Olfers, 1818) & SA & & & cap/foto \\
\hline Chiroderma salvini Dobson, 1878 & SA & & & $\mathrm{col} /$ foto \\
\hline Choeronycteris mexicana Tschudi, 1844 & NA & & A & CZUG \\
\hline Dermanura tolteca (de Saussure, 1860) & MA & & & $\mathrm{col} /$ foto \\
\hline $\begin{array}{l}\text { Desmodus rotundus (É. Geoffroy St.- } \\
\text { Hilaire, 1810) }\end{array}$ & SA & & & cap/foto \\
\hline Glossophaga soricina (Pallas 1766) & SA & & & $\mathrm{col} /$ foto \\
\hline $\begin{array}{l}\text { Leptonycteris yerbabuenae Martínez y Villa- } \\
\text { Ramírez, } 1940\end{array}$ & $\mathrm{AM}$ & & A & $\mathrm{col} /$ foto \\
\hline Macrotus waterhousii Gray, 1843 & MA & & & $\mathrm{col} /$ foto \\
\hline $\begin{array}{l}\text { Sturnira lilium (É. Geoffroy St. Hilaire, } \\
\text { 1810) }\end{array}$ & SA & & & col/foto \\
\hline Sturnira ludovici Anthony, 1924 & SA & & & cap/foto \\
\hline \multicolumn{5}{|l|}{ MORMOOPIDAE } \\
\hline Mormoops megalophylla (Peters, 1864) & $\mathrm{AM}$ & & & $\mathrm{col} /$ foto \\
\hline \multicolumn{5}{|l|}{ MOLOSSIDAE } \\
\hline Nyctinomops femorosaccus (Merriam, 1889) & NA & & & col/foto \\
\hline Nyctinomops macrotis (Gray, 1839) & $\mathrm{AM}$ & & & $\mathrm{col} /$ foto \\
\hline Promops centralis Thomas, 1915 & SA & & & $\mathrm{col} /$ foto \\
\hline $\begin{array}{l}\text { Tadarida brasiliensis (I. Geoffroy Saint- } \\
\text { Hilaire, 1824) }\end{array}$ & $\mathrm{AM}$ & & & $\mathrm{col} /$ foto \\
\hline VESPERTILIONIDAE & & & & \\
\hline
\end{tabular}


Cuadro 1. Continúa.

\begin{tabular}{|c|c|c|c|c|}
\hline & Distribución & Endemismo & NOM-059 & $\begin{array}{l}\text { Medio de } \\
\text { registro }\end{array}$ \\
\hline Eptesicus fuscus (Palisot de Beauvois, 1796) & $\mathrm{AM}$ & & & col/foto \\
\hline Lasiurus blossevillii (Lesson y Garnot, 1826) & $\mathrm{AM}$ & & & $\mathrm{col} /$ foto \\
\hline $\begin{array}{l}\text { Lasiurus cinereus (Palisot de Beauvois, } \\
\text { 1796) }\end{array}$ & $\mathrm{AM}$ & & & $\mathrm{col} /$ foto \\
\hline Lasiurus xanthinus (Thomas, 1897) & NA & & & col/foto \\
\hline $\begin{array}{l}\text { Myotis californicus (Audubon y Bachman, } \\
\text { 1842) }\end{array}$ & AM & & & $\mathrm{col} /$ foto \\
\hline Myotis thysanodes Miller, 1897 & NA & & & $\mathrm{col} /$ foto \\
\hline Myotis velifer (J.A. Allen, 1890) & AM & & & $\mathrm{col} /$ foto \\
\hline Parastrellus hesperus (H. Allen, 1864) & NA & & & col/foto \\
\hline Rhogeessa gracilis Miller, 1897 & MA & EM & & $\mathrm{col} /$ foto \\
\hline \multicolumn{5}{|l|}{ CARNIVORA } \\
\hline \multicolumn{5}{|l|}{ FELIDAE } \\
\hline Lynx rufus (Schreber, 1777) & NA & & & lit/rastro \\
\hline Puma concolor (Linnaeus, 1771 & $\mathrm{AM}$ & & & DVSLP \\
\hline \multicolumn{5}{|l|}{ CANIDAE } \\
\hline Canis latrans Say, 1823 & NA & & & lit/rastro \\
\hline Urocyon cinereoargenteus (Schreber, 1775) & $\mathrm{AM}$ & & & $\begin{array}{l}\text { lit/obs/ } \\
\text { rastro }\end{array}$ \\
\hline \multicolumn{5}{|l|}{ MUSTELIDAE } \\
\hline Mustela frenata Lichtenstein, 1831 & $\mathrm{AM}$ & & & lit/obs \\
\hline \multicolumn{5}{|l|}{ MEPHITIDAE } \\
\hline Conepatus leuconotus (Lichtenstein, 1832) & NA & & & lit/obs \\
\hline Mephitis macroura Lichtenstein, 1832 & $\mathrm{AM}$ & & & lit/obs \\
\hline Spilogale angustifrons Howell, 1902 & $\mathrm{AM}$ & & & lit \\
\hline \multicolumn{5}{|l|}{ PROCYONIDAE } \\
\hline Bassariscus astutus (Lichtenstein, 1830) & NA & & & lit/obs \\
\hline Nasua narica (Linnaeus, 1766) & $\mathrm{AM}$ & & & $\begin{array}{c}\text { lit/ } \\
\text { DVSLP }\end{array}$ \\
\hline Procyon lotor (Linnaeus, 1758) & AM & & & lit/obs \\
\hline \multicolumn{5}{|l|}{ ARTIODACTYLA } \\
\hline \multicolumn{5}{|l|}{ TAYASSUIDAE } \\
\hline Pecari tajacu (Linnaeus, 1758) & AM & & & DVSLP \\
\hline CERVIDAE & & & & \\
\hline
\end{tabular}


Cuadro 1. Continúa.

\begin{tabular}{lccc}
\hline & Distribución & Endemismo & $\begin{array}{c}\text { NOM-059 } \\
\begin{array}{c}\text { Medio de } \\
\text { registro }\end{array}\end{array}$ \\
\hline $\begin{array}{l}\text { Odocoileus virginianus (Zimmermann, } \\
1780)\end{array}$ & AM & $\begin{array}{c}\text { lit/obs/ } \\
\text { rastro }\end{array}$ \\
\hline
\end{tabular}

*especie anteriormente asignada a P. levipes (grupo boylii, ver Álvarez-Castañeda \& González-Ruiz 2009 y Godinez et al. 2011).

de Rodentia (roedores) con 14, Carnivora (carnívoros) con 11, Artiodactyla (pecaríes, venados) y Didelphimorphia (tlacuaches) con dos y Cingulata (armadillos), Lagomorpha (conejos y liebres) y Soricomorpha (musarañas) con una especie cada uno. A nivel de familia las mejor representadas fueron Phyllostomidae con 13 especies y Vespertilionidae con nueve, seguidas de Cricetidae con ocho. Basados en su distribución geográfica actual, 10 especies son endémicas de México y Mesoamérica, 23 son de amplia distribución, 17 compartidas con Norteamérica y nueve con América del Sur (Cuadro 1).

De las 29 especies listadas en el plan de manejo (CONANP 2000), se corroboró la presencia de 23 en el APFFLP. Las otras seis no se incluyeron en el presente escrito debido a que no se capturaron, no se documentó apropiadamente su registro o son especies introducidas (Sorex oreopolus, Sciurus aureogaster, Microtus mexicanus, Puma yagouaroundi, Rattus novergicus y $R$. rattus).

En relación a las especies catalogadas en alguna categoría de riesgo de extinción en la NOM-059-SEMARNAT-2010 tres especies están incluidas: Cratogeomys fumosus, Leptonycteris yerbabuenae y Choeronycteris mexicana (Amenazadas). Las especies endémicas de México resultaron ser ocho: Tlacuatzin canescens, Cratogeomys fumosus, Pappogeomys bulleri, Peromyscus spicilegus, Peromyscus. sp. (grupo boylii, anteriormente asignado a $P$. levipes, ver Álvarez-Castañeda \& González-Ruiz 2009 y Godinez et al. 2011), Sigmodon mascotensis, Artibeus hirsutus y Rhogeessa gracilis (Cuadro 1).

\section{DISCUSIÓN}

El número de mamíferos registrados para el APFFLP se incrementaron en un 156\%, 36 especies más de lo citado previamente y corroborado del plan de manejo (CONANP 2000), y representa el $12 \%$ de la riqueza mastofaunística terrestre de México y $34 \%$ de Jalisco. En relación a su superficie, la riqueza de mamíferos es de 1.9 especies por $10 \mathrm{~km}^{2}$, esto es mayor que en otras ANPs del mismo bioma y con mayor tamaño (Ceballos 2007), por ejemplo para el Nevado de Toluca en el Estado de México, la riqueza es de 0.98 especies por $10 \mathrm{~km}^{2}$ (46 784 ha, 46 especies), San Pedro Mártir, Baja California con 0.63 especies por 10 km² (72 911 ha, 46 especies), 
Sierra La Laguna, Baja California Sur, con 0.38 especies por $10 \mathrm{~km}^{2}$ (112 $437 \mathrm{ha}, 43$ especies), Popo-Izta, Estado de México, con 0.46 especies por $10 \mathrm{~km}^{2}$ (290 284 ha, 42 especies), La Malinche, Estado de México, con 0.87 especies por $10 \mathrm{~km}^{2}(245711$ ha, 40 especies).

Jalisco es reconocido por su riqueza biológica la cual es atribuida a su ubicación en la zona de transición entre la región neártica y neotropical (Iñiguez \& Santana 1993. Guerrero et al. 1995) así como por la influencia de factores topográficos y climáticos (Guerrero et al. 1995, Ramos-Vizcaíno et al. 2007); es posible que la riqueza de mamíferos del APFFLP esté influenciada por la ubicación en este punto de transición dentro del estado, lo que puede reflejarse al considerar la distribución de sus especies, ya que el 39\% (23) exhiben una distribución extensa, el 29\% (17) son compartidas con Norteamérica, el 17\% (10) son endémicas de México y Mesoamérica y el 15\% (9) compartidas con América del Sur. Similar a lo reportado en el área de Arcediano (con bioma tropical, ubicada a $30 \mathrm{~km}$ NE del APFFLP) con el 29\% (15) de sus especies de amplia distribución, 27\% (14) compartidas con Norteamérica, 23\% (12) con América del Sur y 21\% (11) son endémicas de México y Mesoamérica (Godinez et al. 2008). Esto contrasta con la zona de Chamela-Cuixmala, una ANP de bioma tropical ubicada en la costa de Jalisco, en la que el 34\% (24) son compartidas con América del Sur, 30\% (21) son endémicas de México y Mesoamérica, 26\% (18) son de amplia distribución y apenas el 10\% (7) con Norteamérica (Miranda 2002).

El orden con mayor número de especies fue Chiroptera con 27, representando el 46\% de los mamíferos del ANP, esto es, el 37\% de los murciélagos citados para el estado (Godinez et al. 2011). En segundo lugar, el orden Rodentia con 14 especies representa el $24 \%$ de los mamíferos del ANP y $23 \%$ de los roedores de Jalisco (Godinez et al. 2011), fue el único orden con más especies (14) que las áreas de Chamela-Cuixmala (13) y Arcediano (13). A nivel de familia sobresalen Vespertilionidae (murciélagos) con nueve especies, Sciuridae (ardillas) con tres y Geomyidae (tuzas) con dos, más especies que Chamela-Cuixmala $(5,1,1$, respectivamente) y Arcediano $(4,2,0$, respectivamente).

De los endemismos registrados (8) corresponden al 5\% de los del país y 14\% de la mastofuna del ANP, esto es mayor que lo reportado para Maderas del Carmen, en dónde el 3\% (2) de sus especies son endémicas a México; San Pero Mártir con el 4\% (2); Sierra La Laguna 7\% (3); y La Malinche 12.5\% (5) (Ceballos 2007); aunque fue menor en comparación a las Reservas de la Biosfera Chamela-Cuixmala (Miranda 2002) y Sierra de Manantlán (INE 2000), en el que el 26\% (18) y 16\% (18) de su mastofauna respectivamente, es endémica a México, sin embargo resalta que de los endemismos presentes en el APFFLP, dos especies Peromyscus sp. (grupo boylii) y Rhogeessa gracilis, no se incluyen en las ANPs antes mencionadas.

A pesar de que la riqueza del APFFLP no fue mayor que la conocida para las Reservas de la Biosfera Chamela-Cuixmala (Miranda 2002) y Sierra de Manantlán 
(INE 2000), si sobresale lo aquí registrado, ya que esta área incorpora especies que no se citan para las otras ANPs de Jalisco. Estas especies son: Ictidomys mexicanus, Sciurus nayaritensis, Peromyscus sp. (grupo boylii), Peromyscus maniculatus, Criptotis parva, Nyctinomops femorosaccus, N. macrotis y Rhogeessa gracilis, lo que incrementa en un 5\% las especies de Jalisco incluidas en alguna área de protección, además, es muy probable que $R$. gracilis, especie endémica a México, se documenta por primera vez en una ANP del país.

En relación a la riqueza de mamíferos (152) reportada para la FVT por Escalante et al. (2007), la mastofauna del APFFLP representa el 39\% del total, el 69\% (11) de los carnívoros, $57 \%$ (27) de los quirópteros y el 21\% (14) de los roedores. Así mismo, los endemismos (8) y las especies en riesgo (3) del APFFLP son el $18 \%$ y $10 \%$ respectivamente, de los mamíferos endémicos (45) y en riesgo (31) dentro de la FVT. Se ha considerado que los factores que mejor explican la distribución de los mamíferos dentro de la FVT son la altitud y la vegetación, siendo rara la presencia de murciélagos por arriba de los 1500 msnm (Gámez et al. 2012). Sin embargo, en el presente trabajo, de las 27 especies de murciélagos documentadas, 18 fueron capturadas a 1849 msnm, algunas con abundancia considerable (ej.: Myotis californicus con 56 capturas, Tadarida brasiliensis 51, Macrotus waterhousii 46 y Eptesicus fuscus 22). Por tanto, la riqueza aquí reportada (59) significó dos especies más que la esperada por Escalante et al. (2007) para la celda en dónde se ubica el APFFLP, esto confirma la necesidad y relevancia de estudios puntuales para corroborar las predicciones de la distribución de los mamíferos basados en modelos de nicho ecológico.

En conclusión el APFFLP no ha sido considerada una área de protección relevante (Fa \& Morales 1991, Escalante 2003, Ceballos 2007, Vázquez \& Valenzuela-Galván 2009), ni área prioritaria de conservación (Arriaga et al. 2000). Sin embargo aquí se demuestra su importancia, por su riqueza de especies de mamíferos y el considerable número de endemismos presentes en comparación con otras áreas relevantes en el país.

Agradecimientos. A la Universidad de Guadalajara y PROMEP por el financiamiento otorgado en el proyecto "Fauna Urbana y Periurbana de Jalisco: Diversidad y Ecología"UGD-CA-51. A Andreola Velleryd, Martha R. Gallardo, Uriel Jiménez y Arquímedes Godoy, por su importante apoyo en el trabajo de campo. A Gerardo Cabrera y Karina Aguilar de la Dirección de Conocimiento y Vida Silvestre del APFFLP por las facilidades para el desarrollo de este trabajo. A José Luis Navarrete-Heredia y a dos revisores anónimos por sus valiosos comentarios al manuscrito.

\section{LITERATURA CITADA}

Álvarez, T., Álvarez-Castañeda, S. T. \& López-Vidal, J. C. 1994. Claves para murciélagos mexicanos. Centro de Investigaciones Biológicas del Noreste, S.C. y Escuela Nacional de Ciencias Biológicas I.P.N. La Paz, BCS. 
Álvarez-Castañeda, S. T. \& González-Ruiz, N. 2009. Peromyscus levipes (Rodentia: Cricetidae). Mammalian species, 824: 1-6.

Aranda, M. 2000. Huellas y otros rastros de los mamíferos grandes y medianos de México. Instituto de Ecología, A.C., Xalapa, México.

Arriaga, L., Espinoza, J. M., Aguilar, C., Martínez, E., Gómez, L. \& Loa, E. (Coords.). 2000. Regiones terrestres prioritarias de México. Escala de trabajo 1:1 000 000. Comisión Nacional para el Conocimiento y uso de la Biodiversidad, México.

Ceballos, G. \& Navarro, D. 1991. Diversity and conservation of Mexican mammals, pp. 167-198. In: M. A. Mares \& D. J. Schmidly (Eds.). Topics in Latin American mammalogy: history, biodiversity, and education. University of Oklahoma Press, Norman. USA.

Ceballos, G. 2007. Conservation priorities for mammals in megadiverse Mexico: the efficiency of reserve networks. Ecological Applications, 17: 569-578.

Ceballos, G., Arroyo-Cabrales, J. \& Medellín, R. A. 2002. The mammals of México: composition, distribution and status. Occasional Papers of the Museum of Texas Tech University, No. 218.

Cervantes, F. A., Matamoros-Trejo, G. \& Martínez-Mateos, I. 1995. Mamíferos silvestres de la unidad de evaluación y monitoreo de la biodiversidad "Ing. Luis Macías Arellano", San Cayetano, Estado de México. Anales del Instituto de Biología, Serie Zoología, 66: 233-239.

CONABIO-CONANP-TNC-PRONATURA-FCF, UANL. 2007. Análisis de vacios y omisiones en conservación de la biodiversidad terrestre de México: espacios y especies. Comisión Nacional para el Conocimiento y Uso de la Biodiversidad, Comisión Nacional de Áreas Naturales Protegidas. The Nature Conservancy-Programa México, Pronatura, A.C., Facultad de Ciencias Forestales, Universidad Autónoma de Nuevo León, México.

CONANP (Comisión Nacional de Áreas Naturales Protegidas). 2000. Programa de manejo del Área de Protección de Flora y Fauna La Primavera México. CONANAP-SEMARNAT. México D.F.

CONANP (Comisión Nacional de Áreas Naturales Protegidas). 2012. Áreas protegidas decretadas. http://www.conanp.gob.mx (consultado el 23 de junio de 2013).

Escalante, T. 2003. Determinación de prioridades en las áreas de conservación para los mamíferos terrestres de México, empleando criterios biogeográficos. Anales del Instituto de Biología, Serie Zoología, 74: 211-237.

Escalante, T., Rodríguez, G., Gámez, N., León-Paniagua, L., Barrera, O. \& Sánchez-Cordero, V. 2007. Biogeografía y conservación de los mamíferos, pp. 485-502. In: I. Luna, J. J. Morrone \& D. Espinosa (Eds.). Biodiversidad de la Faja Volcánica Transmexicana. UNAM, México, D.F.

Fa, J. E. \& Morales, L. M. 1991. Mammals and protected areas in the Trans-Mexican Neovolcanic Belt, pp 199- 226. In: M. L. Mares \& D. J. Schmidly (Eds.). Latin American mammalogy, history, biodiversity and conservation. University of Oklahoma Press, Norman. U.S.A.

Fuller, T., Munguía, M., Mayfield, M., Sanchez-Cordero, V. \& Sarkar, S. 2006. Incorporating connectivity into conservation planning: A multi-criteria case study from central México. Biological Conservation, 133: 131-142.

Gámez, N., Escalante, T., Rodríguez, G., Linaje, M. \& Morrone, J. J. 2012. Caracterización biogeográfica de la Faja Volcánica Transmexicana y análisis de los patrones de distribución de su mastofauna. Revista Mexicana de Biodiversidad, 83: 258-272.

Godínez, E. G., Del Río, A. E., Covarrubias, H. O., López, R. \& Cortés, J. 2008. Guía de mamíferos de Arcediano. Gobierno del estado de Jalisco, Comisión Estatal del Agua.

Godínez, E. G., González-Ruiz, N. \& Ramírez-Pulido, J. 2011. Lista actualizada de los mamíferos de Jalisco, México: implicaciones de los cambios taxonómicos. Therya, 2: 7-35.

Guerrero, S. \& Cervantes, F. A. 2003. Lista comentada de los mamíferos terrestres del estado de Jalisco, México. Acta Zoológica Mexicana (n. s.), 89: 93-110. 
Guerrero, S., Téllez, J. \& Salido, R. A. 1995. Los mamíferos de Jalisco: análisis zoogeográfico. Biotam, 6: 13-30.

Hall, E. R. 1981. The mammals of North America. John Wiley and Sons, New York. 1175 pp.

Hockings, M. 2003. Systems for assessing the effectiveness of management in protected areas. Bioscience, 53: 823-832.

INE (Instituto Nacional de Ecología). 2000. Programa de manejo de la reserva de la biosfera Sierra de Manantlán. México. INE- SEMARNAT, México D.F.

Iñiguez, D. L. 1993. Patrones ecológicos de la comunidad de murciélagos de la Sierra de Manantlán, Jalisco, pp. 355-370. In: R. Medellín \& G. Ceballos (Eds.). Avances en el estudio de los mamíferos de México. Asociación Mexicana de Mastozoología, A.C. Publicaciones Especiales, vol. 1. México, DF.

Iñiguez, D. L. \& Santana, E. 1993. Patrones de distribución y riqueza de especies de los mamíferos del occidente de México, pp. 65-86. In: R. Medellín \& G. Ceballos (Eds.). Avances en el estudio de los mamiferos de México. Asociación Mexicana de Mastozoología, A.C. Publicaciones Especiales, vol. 1. México, DF.

Jones, J. K. Jr., Gemoways. H. H. \& Watkins. L. C. 1970. Bats of the genus Myotis from western Mexico, with a key to species. Transactions of the Kansas Academy of Science, 73: 409-418.

Juárez-Aguilar, A. 1995. Los carnívoros (clase Mammalia: orden Carnivora) del bosque La Primavera, Jalisco, su distribución, abundancia y diversidad por tipos de vegetación. Tesis de Licenciatura, Universidad de Guadalajara. $118 \mathrm{pp}$.

Kumirai, A. \& Jones Jr., J. K. 1990. Nyctinomops femorosaccus. Mammalian Species, 349: 1-5.

Medellín, R. A., Arita, H. T. \& Sánchez, O. 2008. Identificación de los murciélagos de México, clave de campo. 2 da edición. Instituto de Ecología, UNAM, México D.F.

Miranda, A. 2002. Diversidad, historia natural, ecología y conservación de los mamíferos de Chamela, pp. 359-377. In: F A. Noguera, J. H. Vega, A. N. García \& M. Quesada (Eds.). Historia natural de Chamela. Instituto de Biología, UNAM.

Morrone, J. J. 2005. Hacia una síntesis biogeográfica de México. Revista Mexicana de Biodiversidad, 76: 207-252.

Ramamoorthy, T. P., Bye, R., Lot, A. \& Fa, J. (Eds). 1998. Diversidad biológica de México: orígenes $y$ distribución. Instituto de Biología, UNAM, México, D.F.

Ramírez-Pulido, J., Arroyo-Cabrales, J. \& Castro-Campillo, A. 2005. Estado actual y relación nomenclatural de los mamíferos terrestres de México. Acta Zoológica Mexicana (n. s.), 21: 21-82.

Ramos-Vizcaíno, I., Guerrero, S. \& Huerta, F. M. 2007. Patrones de distribución geográfica de los mamíferos de Jalisco, México. Revista Mexicana de Biodiversidad, 78: 175-189.

Rzedowski, J. 1978. Vegetación de México. Ed. Limusa. México.

Segura-Trujillo, C.A. \& Navarro-Pérez, S. 2010. Escenario y problemática de conservación de los murciélagos (Chiroptera) cavernícolas del Complejo Volcánico de Colima, Jalisco-Colima. Therya, 1: $189-206$.

SEMADET (Secretaría de Medio Ambiente y Desarrollo Territorial). 2013. Áreas Naturales protegidas en Jalisco. http://www.jalisco.gob.mx/semadet (consultado el 23 de junio de 2013).

SEMARNAT (Secretaría de Medio Ambiente y Recursos Naturales). 2010. Protección ambiental-Especies nativas de México de flora y fauna silvestres-Categorías de riesgo y especificaciones para su inclusión, exclusión o cambio- Lista de especies en riesgo. Diario Oficial de la Federación, jueves 30 de diciembre de 2010, 1: 1-77.

Vázquez, L. B \& Valenzuela-Galván, D. 2009. ¿Qué tan bien representados están los mamíferos mexicanos en la red federal de áreas naturales protegidas del país? Revista Mexicana de Biodiversidad, 80: 249-258. 
Vázquez, L. B., Medellín, R. A. \& Cameron, G. N. 2000. Population and community ecology of small rodents in montane forest of Western Mexico. Journal of Mammalogy, 81: 77-85.

Velázquez. A., Mas, J. F., Díaz, J. R., Mayorga, R., Alcántara, P. C., Castro, R., Fernández, T., Bocco, G., Ezcurra, E. \& Palacio, J. L. 2002. Patrones y tasas de cambio de uso de suelo en México. Gaceta Ecológica No. 62. Secretaría de Medio Ambiente y Recursos Natrales México. 